\title{
Fine temporal structure of beta-band synchronization in Parkinson's disease: experiments, models and mechanisms
}

\author{
Leonid L Rubchinsky ${ }^{1,2^{*}}$, Choongseok Park ${ }^{1}$, Robert M Worth ${ }^{3}$ \\ From Nineteenth Annual Computational Neuroscience Meeting: CNS*2010 \\ San Antonio, TX, USA. 24-30 July 2010
}

Synchronous oscillatory activity in the beta frequency band is a characteristic feature of neuronal dynamics of basal ganglia in PD and is hypothesized to be related to disease's hypokinetic symptoms [1]. However the character and nature of this activity is very much opened question. The dynamics of these oscillations and their mechanisms are the subjects of this study. We simultaneously record spikes and LFP from subthalamic nucleus (STN) of patients with PD and analyze the phase locking between these signals as it develops in time in order to explore the variability of beta-band oscillations in BG. We then match the experimental results with the models of BG networks. STN LFP has mostly synaptic origin and (given the absence of lateral connections within STN) is representative of input signals to STN. The fragile temporal structure of this synchrony necessitates the use of time-sensitive data analysis methods, so we followed our earlier developments $[2,3]$ to detect oscillatory episodes with synchronous dynamics.

To recover the fine temporal structure of these episodes we constructed the first-return maps for the difference of the phases of oscillations and quantified the transition between different regions of the maps. Thus we were able to identify the succession of synchronized and desynchronized periods within oscillatory episodes with overall tendency for synchrony. The synchronized dynamics is interrupted by essentially nonsynchronized periods. These desynchronization events are distributed in a specific way - there is a predominance of short desynchronization events. The signals go out of phase

\footnotetext{
* Correspondence: leo@math.iupui.edu

'Department of Mathematical Sciences and Center for Mathematical Biosciences Indiana University Purdue University Indianapolis, Indianapolis, IN 46202, USA
}

for just one cycle of oscillations more often than for two or a larger number of cycles. An alternative scenario (desynchronization events are longer but less frequent) would produce the same degree of average synchrony. However our results show that this alternative is not realized in the parkinsonian BG.

To understand the mechanisms of the observed synchronous activity, we developed conductance-based models for subthalamo-pallidal circuits (based on [4]). The model retains substantial amount of biological realism (both, membrane properties and anatomical organization of the inhibitory-excitatory network) and exhibits synchronous oscillatory patterns. We studied the model dynamics under the variation of parameters, which are modulated by dopamine and subjected the model output to the same data-analysis techniques, as we used for experimental data. Thus we identified the parameter domain, where the model dynamics and experimentally observed dynamics are similar in how the synchronized patterns evolve in time. This area is on the boundary of fully synchronous regime and is also characterized by a proximity to a non-synchronized (and presumably healthy) dynamics.

The realistic patterns arise in the model network, when synaptic projections (normally suppressed by dopamine) become stronger. The intermittent synchrony in the parkinsonian state may be a result of a propensity of BG circuits to be engaged in the brief synchronized episodes of activity needed for movement control. The low-dopamine state with stronger coupling may result in a partial suppression of this very transient (and hardto-detect) character of neuronal dynamics, favoring only short desynchronization events, which interrupt mostly synchronous episodes. 


\section{Acknowledgements}

Supported by the National Institutes of Health grant 1R01NS067200 (NSF/ $\mathrm{NIH}$ CRCNS program).

\section{Author details}

'Department of Mathematical Sciences and Center for Mathematical Biosciences Indiana University Purdue University Indianapolis, Indianapolis, IN 46202, USA . ${ }^{2}$ Stark Neurosciences Research Institute, Indiana University School of Medicine, Indianapolis, IN 46202, USA . ${ }^{3}$ Department of

Neurological Surgery, Indiana University School of Medicine, Indianapolis, IN 46202, USA.

Published: 20 July 2010

\section{References}

1. Hammond C, Bergman H, Brown P: Pathological synchronization in Parkinson's disease: networks, models and treatments. Trends Neurosci 2007, 30:357-364.

2. Hurtado JM, Rubchinsky LL, Sigvardt KA: Statistical method for detection of phase locking episodes in neural oscillations. J Neurophysiol 2004, 91:1883-1898.

3. Hurtado JM, Rubchinsky LL, Sigvardt KA, Wheelock VL, Pappas CTE: Temporal evolution of oscillations and synchrony in GPi/muscle pairs in Parkinson's disease. J Neurophysiol 2005, 93:1569-1584.

4. Terman D, Rubin JE, Yew AC, Wilson CJ: Activity patterns in a model for subthalamopallidal network of basal ganglia. J Neurosci 2002 22:2963-2976.

doi:10.1186/1471-2202-11-S1-O6

Cite this article as: Rubchinsky et al: Fine temporal structure of betaband synchronization in Parkinson's disease: experiments, models and mechanisms. BMC Neuroscience 2010 11(Suppl 1):O6.

\section{Submit your next manuscript to BioMed Central} and take full advantage of:

- Convenient online submission

- Thorough peer review

- No space constraints or color figure charges

- Immediate publication on acceptance

- Inclusion in PubMed, CAS, Scopus and Google Scholar

- Research which is freely available for redistribution

Submit your manuscript at www.biomedcentral.com/submit 